\title{
Mucormicosis en pacientes diabéticos post infección por COVID-19
}

\author{
Mucormycosis in diabetic patients \\ post-COVID-19 infection
}

\author{
Federico Elguera-Falcón ${ }^{1,}$ \\ Rober Cumpa-Quirózz
}

Elguera-Falcón F., Cumpa-Quiróz R, Mucormicosis en pacientes diabéticos post infección por COVID-19. Rev Soc Peru Med Interna. 2020;33(4): 176-182.

https://doi.org/10.36393/spmi.v33i4.568

\begin{abstract}
RESUMEN
La mucormicosis es una infección causada por hongos del orden Mucorales. Entre los factores de riesgo más comunes están la diabetes mellitus no controlada y complicada con cetoacidosis diabética, uso de corticoides, etc. Las características clínicas dependen del tipo de compromiso, siendo las más frecuentes: fiebre, sinusitis, proptosis, cefalea, parálisis de nervios craneales, etc. El tratamiento consta de terapia antifúngica temprana, cirugía precoz y amplia, y manejo adecuado de las comorbilidades. Se describe el cuadro clínico de cuatro pacientes diabéticos descompensados que presentaron mucormicosis rino-órbito-cerebral posterior a una infección COVID-I9.
\end{abstract}

Palabras claves: Mucormicosis, diabetes mellitus, cetoacidosis diabética, sinusitis, COVID-I9.

\begin{abstract}
Mucormicosis is an infection caused by Mucorales fungi. Among the most common risk factors are uncontrolled and complicated diabetes mellitus with diabetic ketoacidosis, corticoid use, etc. Clinical characteristics depend on the type ofinvolvement, the most common being: fever, sinusitis, proptosis, headache, paralysis of cranial nerves, etc.

Treatment consists of early antifungal therapy, early and extensive surgery, and proper management of comorbilities. The clinical picture of four decompensated diabetic patients who had rhino-orbit-brain mucormycosis following a COVID-19 infection is described.
\end{abstract}

Keywords: Mucormicosis, diabetes mellitus, diabetic ketoacidosis, sinusitis, COVID-19.

\section{INTRODUCCIÓN}

La mucormicosis es una infección angioinvasiva causada por hongos del orden Mucorales, clase Zigomicetos. Es la tercera micosis invasiva en orden de importancia luego de

1 Médico internista. Hospital nacional Arzobispo Loayza, Lima, Perú.

2 Médico internista. Facultad de Medicina, Universidad Peruana Cayetano Heredia. Hospital Nacional Arzobispo Loayza, Lima, Perú. las infecciones causadas por Candida spp. y Aspergillus $s p p$, presentándose como infecciones oportunistas y graves, de evolución rápida o menos frecuentemente crónicas con curso indolente. Los géneros más importantes son Rhizopus, Mucor, Absidia y Cunninghamella (1-7), siendo R. arrhizus (denominado también $R$. oryzae), el más comúnmente hallada en humanos (50-60\% de casos) (2,5-8). En pacientes inmunocomprometidos, la mayoría se presenta en pacientes con cuadros de cetoacidosis diabética, o teniendo como antecedentes enfermedades hematológicas, trasplante de órganos sólidos o trasplante de células madre $(1-6)$. Tiene una incidencia anual estable de 1,7 casos por millón $(5,6)$. A continuación, se describe el cuadro de cuatro pacientes con mucormicosis posterior a infección COVI-19.

\section{PRESENTACIÓN DE LOS CASOS}

\section{CASO 1}

Paciente varón de 45 años de edad, hipertenso y diabético de larga evolución, mal controlado, usuario irregular de losartán, insulina y metformina. Acudió por cuadro de 15 días de evolución caracterizado por malestar general y fiebre. Un médico particular prescribió paracetamol y azitromicina, y al no tener mejoría agregó ceftriaxona y dexametasona. Al persistir el cuadro y bajar la saturación de oxígeno, acudió al hospital de Ate, el 11/08/20, donde 
fue diagnosticado de neumonía COVID-19 e insuficiencia respiratoria. En el examen preferencial describieron leve incremento de volumen en la hemicara derecha y uso de músculos accesorios respiratorios. En su evolución presentó hemiparesia izquierda, proptosis y eritema ocular derechos, y lesión ulcerada en paladar duro derecho de 3 x $1 \mathrm{~cm}$. Se realizó una TAC (13/08/20) donde se evidenció imágenes hipodensas en el lóbulo temporal y protuberancial derecha, sugerentes de infarto agudo. En la TAC de control (02/09/20) se observó un absceso cerebral que se extiendía desde la fosa posterior afectando el hemisferio derecho del cerebelo y protuberancia, con recorrido anterior transtentorial hacia el lóbulo temporal, pansinusitis y mastoiditis derecha. Fue trasferido a este hospital el 10/09/20 para manejo quirúrgico. Con la presunción diagnóstica de mucormicosis rino-órbito-cerebral se inició tratamiento con anfotericina $\mathrm{B}$ y luego isavuconazol. El paciente solicitó retiro voluntario. Figuras 1 y 2.

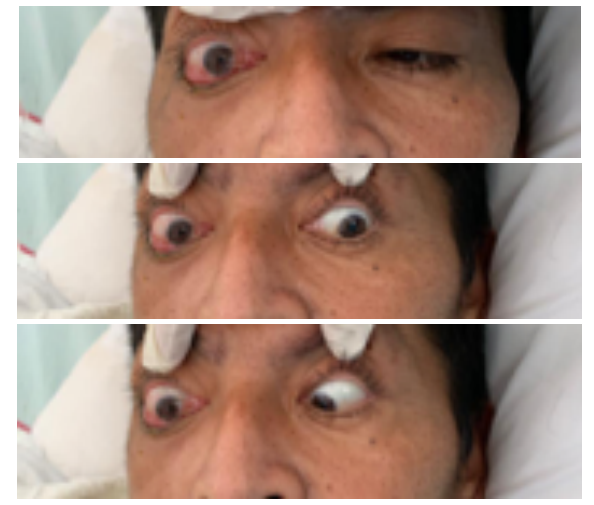

Figura I. Proptosis, quemosis y parálisis de músculos óculo motores ojo derecho.

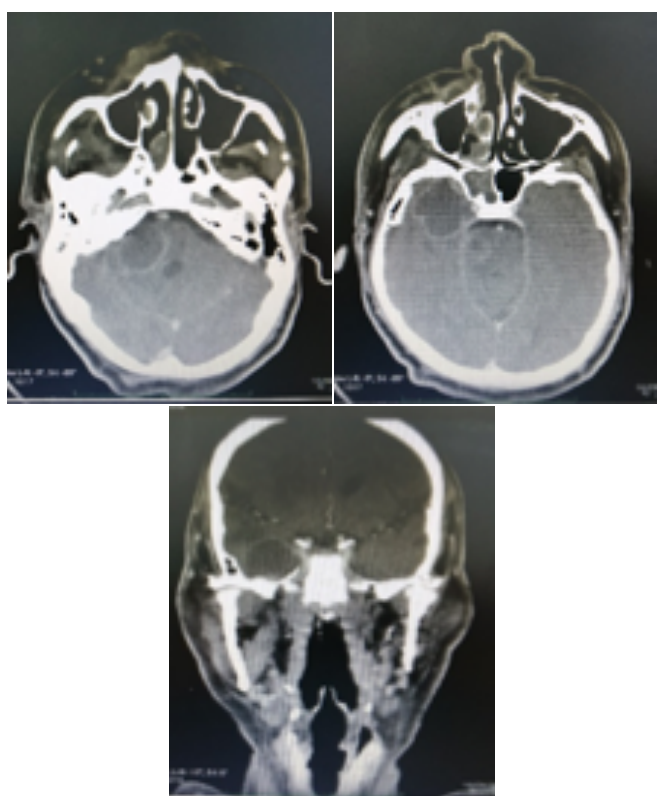

Figura 2. TAC: absceso cerebral que se extiende desde la fosa posterior afectando el hemisferio derecho del cerebelo y protuberancia, con recorrido anterior transtentorial hacia el lóbulo temporal, pansinusitis y mastoiditis derecha

\section{CASO 2}

Varón 51 años, procedente de Cañete, maquinista, diabético de larga data, usuario de metformina. Diagnosticado de COVID-19 en junio de este año, recibió tratamiento que incluía corticoides en forma ambulatoria. Acudió al servicio de emergencia el 078/8/20, por tiempo de enfermedad de dos meses, por secreción nasal purulenta, edema periorbitario y secreción purulenta por el ojo izquierdo y aumento volumen de la hemicara izquierda. En el examen físico se encontró aumento volumen hemicara izquierda, parálisis facial izquierda, quemosis, edema palpebral y periorbitario y pérdida de visión del ojo izquierdo; además, presencia de costras en tabique y meatos. El 29/08/20 se realizó cirugía endoscópica nasosinusal con antrostomía, etmoidectomía, drenaje de seno maxilar y apertura de senos frontal y esfenoidal, tomando biopsias las que se reportaron compatibles con mucormicosis. Se inició tratamiento primero con anfotericina B y luego con isovuconazol. El 16/09/20, el paciente reingresó a sala de operaciones donde se le realizó una craneotomía con exéresis órbitonaso-sinusal, evidenciando tejido óseo necrótico friable, purulento en la región frontal. La TAC (26/09/20) mostró signos sugerentes de mucormicosis de senos paranasales frontal, esfenoidal y etmoidal izquierdos. La evolución fue favorable y salió de alta con tratamiento de isavuconazol por vía oral. Diagnóstico final: mucormicosis rino-órbitocerebral. Figuras 3 y 4.

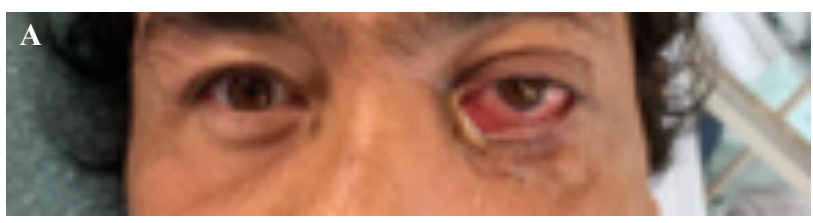

Figura 3. Aumento de volumen de la hemicara izquierda, con parálisis facial, quemosis, edema palpebral y periorbitario izquierdo.

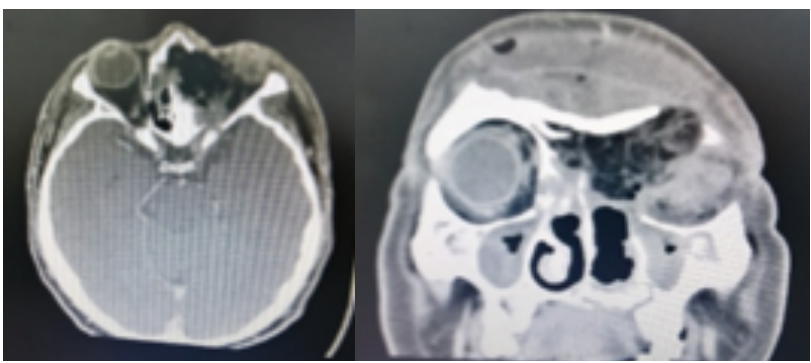

Figura 4. TAC: control postquirúrgico, pansinusitis

\section{CASO 3}

Paciente varón, de 45 años, natural de Jaén, Cajamarca, zapatero. Diagnóstico reciente de diabetes mellitus un mes antes, sin control médico. Contacto con paciente COVID-19 hace un mes, recibió medicinas que no recuerda. Acudió al hospital el 18/09/20, con un tiempo de enfermedad de tres semanas, caracterizado por obstrucción nasal, edema y eritema del ojo izquierdo, acompañado de secreción serohemática, dolor y fiebre. Al examen físico se 
evidenció edema y eritema del párpado superior izquierdo, aumento de volumen de la hemicara izquierda a predominio periorbitario con secreción verdosa amarillenta, proptosis, quemosis, presencia de tejido necrótico en el borde interno del globo ocular izquierdo, pupila izquierda poco reactiva a la luz y limitación de los movimientos oculares. Un informe de TAC (15/09/20): componente blando con compromiso de los músculos extrínsecos, protrusión del globo ocular; lesión hipodensa frontal izquierda con edema periférico y pansinusitis. TAC de control (19/9/20): lesión de densidad de tejidos blandos que ocupa el seno maxilar izquierdo, y los senos esfenoidales y frontales. Absceso frontal izquierdo con edema cerebral periférico que desplaza la línea media y comprime el asta lateral del ventrículo lateral. Ingresó a sala de operaciones el 20/09/20 donde se encuentra necrosis de mucosa septal bilateral, ausencia de cornete medio izquierdo y secreción purulenta, realizándose exéresis de tejido necrótico de pared lateral y septo nasal izquierdo, aspiración de secreción purulenta del seno maxilar izquierdo. Biopsia nasal (22/09/20): muestra constituida por tejido necrótico. El 30/09/20 reingresó a
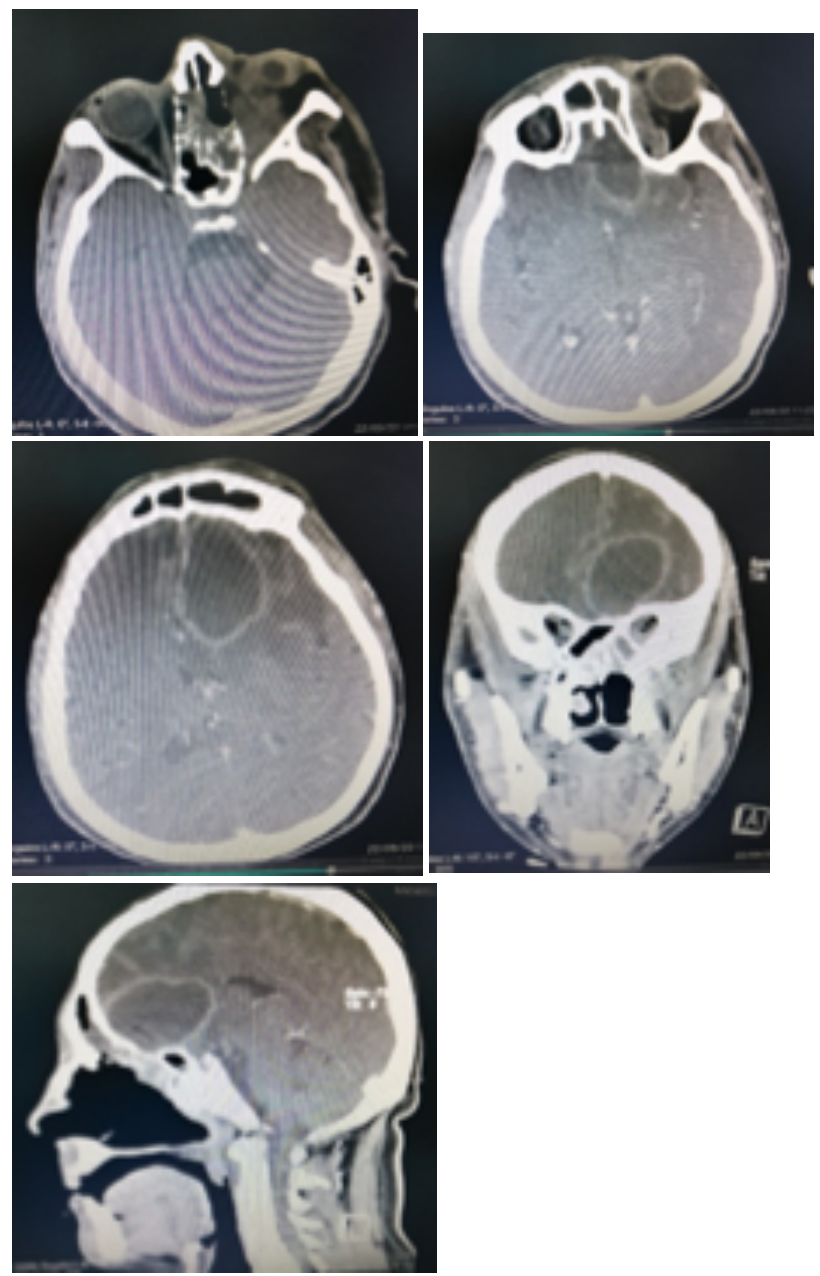

Figura 5. TAC: lesión que ocupa seno maxilar izquierdo, senos esfenoidales y frontales. Absceso frontal izquierdo con edema cerebral periférico que desplaza línea media y comprime asta lateral del ventrículo lateral sala de operaciones y se evidenció presencia de absceso intracerebral, el cual se drenó y se envió muestra para cultivo. El paciente fue intervenido en tres ocasiones por equipo quirúrgicos de diversas especialidades. Recibió anfotericina B y luego isovuconazol. Diagnóstico final: mucormicosis rino-órbito- cerebral. Figura 5.

\section{CASO 4}

Paciente mujer de 48 años con el diagnóstico de diabetes mellitus desde hace un año y en tratamiento con metformina $850 \mathrm{mg}$ cada 8 horas. Infección COVID-19 hace un mes, recibió tratamiento ambulatorio que no recordaba. También, hace un mes presentó dolor dental y le realizaron exodoncias. Ingresó el 25/09/20 por cuadro de tres semanas de evolución caracterizado por presentar lesión proliferativa de coloración negra en el paladar, dolorosa y que luego de una semana se incrementó el tamaño de la lesión, presentando secreción purulenta y se agregó dolor en la hemicara izquierda. Al examen: lesión ulcerada y necrótica en el paladar duro de 1,5 cm de diámetro, no sangrante, con secreción purulenta y aumento de volumen de la hemicara izquierda; dientes en mal estado de conservación e higiene, absceso gingival. Evaluada por otorrinolaringología, se evidenció tejido necrótico y perforación septal, con solución de continuidad en el paladar duro. Se realizó biopsia el 25/09/20 confirmándose cambios histológicos y presencia de hifas compatibles con mucormicosis. La tomografía del macizo facial (23/09/20) mostró imágenes óseas permeativas del reborde alveolar superior izquierdo

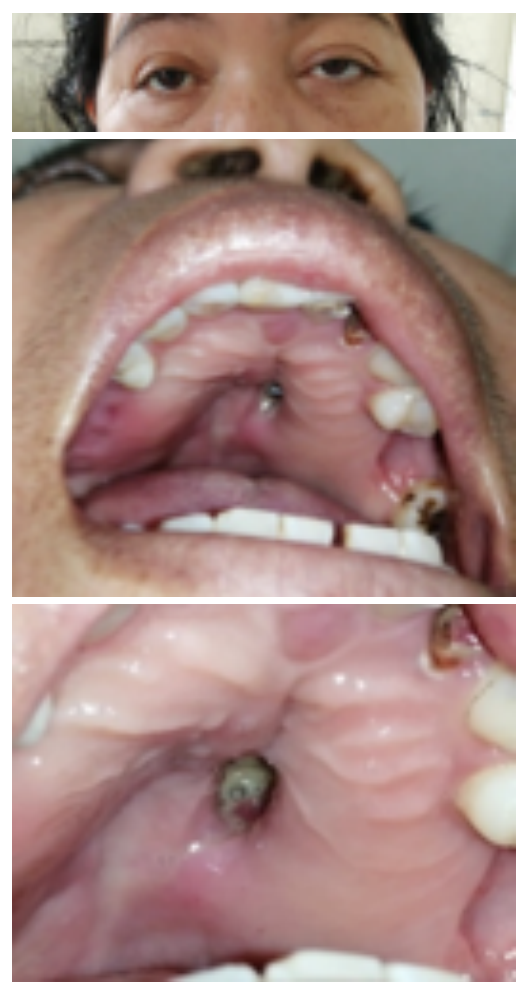

Figura 6. aumento de volumen hemicara izquierda, ptosis palpebral izquierda, lesión ulcero necrótica en paladar, con secreción purulenta; dientes en mal estado de conservación 
con extensión a las paredes anterior y posterior seno maxilar izquierdo; y, pansinusitis. TAC cerebral: no lesiones intraparenquimales. Recibió tratamiento con anfotericina $\mathrm{B}$ y limpieza quirúrgica. Figura 6 y 7.

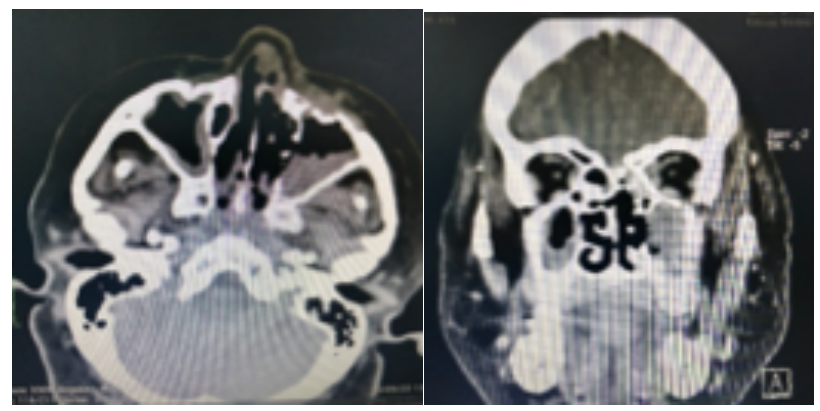

Figura 7. TAC: pansinusitis

\section{DISCUSIÓN}

Los mucorales son hongos saprófitos que se encuentran en materia en descomposición como pan, frutas, hojas muertas, abono vegetal, excremento de animales, tierra rica en carbohidratos etc. ${ }^{2,5,9}$ Además, pueden ser aislados del medio hospitalario (renovación de nosocomios, uso de bandas elásticas y equipos de monitoreo contaminados). Se encuentran en $3 \%$ de muestras de esputo y $1 \%$ en heces en adultos sanos, y puede encontrarse también en el $22 \%$ de muestras de aire dentro de un nosocomio comparado con 5 $\%$ del ambiente exterior. ${ }^{9}$

Los mucorales tienen gran afinidad por las arterias y se adhieren a su pared, crecen a lo largo de la lámina elástica interna de los vasos sanguíneos causando trombosis, isquemia y necrosis. ${ }^{5}$

Los mecanismos de ingreso al hospedero son: ${ }^{3-5,8-10}$

1. Diseminación por vía aérea: los mucorales se implantan en la mucosa nasal, oral o conjuntival a partir de las cuales se inicia la invasión a los tejidos provocando trombosis, isquemia, necrosis, perforación de tabique nasal y extensión a senos paranasales, región orbitaria, conducto nasolacrimal y finalmente al sistema nervioso central o continúan con diseminación hematógena. Además, a partir de los cornetes pueden ser inhalados al interior de los alvéolos.

2. Inoculación directa: por pérdida de la integridad de la piel, secundaria a cirugías, quemaduras u otros traumatismos o accesos endovenosos (catéteres contaminados o usuarios de drogas).

3. Adquisición por ingestión.

4. Luego de una extracción dental.

Los mucorales pueden colonizar mucosas, pero no producen ningún tipo de infección o invasión a menos de que haya un factor que predisponga al hospedero. ${ }^{6}$ No se ha reportado propagación de persona a persona. ${ }^{11}$ Factores de riesgo: ${ }^{1,4-6,8,9,12}$

1. Alteraciones en la fagocitosis. Los pacientes diabéticos tienen alteraciones en el sistema inmunológico, tanto a nivel celular (disminución de actividad fagocítica y fungicida de los neutrófilos e incapacidad para la opsonización) como humoral.

2. Cetoacidosis: Los organismos Rhizopus tienen una enzima, la cetona reductasa, que les permite mantenerse en glicemias altas y estados ácidos. La cetoacidosis además disminuye la permeabilidad de la barrera hematoencefálica contribuyendo a la predilección por la enfermedad rinocerebral en estos pacientes. Rhizopus oryzae y Mucor sp. presentan crecimiento óptimo en medios con temperatura de $39^{\circ} \mathrm{C}, \mathrm{pH}$ ácido y alto contenido de glucosa. Otras entidades productoras de acidosis pueden también predisponer a la infección, tal como sepsis, uremia.

3. La interacción entre esporas, hierro y transferrina pueden determinar la tasa de crecimiento del hongo. El hierro que se separa de la transferrina (estimulado por el $\mathrm{pH}$ ácido) puede ser utilizado para su crecimiento. El uso de desferroxiamina (quelante que revierte la actividad fungistática del suero) incrementa la proliferación de los Mucorales y mortalidad.

4. Sistemas activo cetona-reductasa: permite al hongo crecer en un medio ácido, rico en glucosa y con baja tensión de oxígeno.

5. Otras condiciones también son factores de riesgo: neutropenia, disfunción de neutrófilos, cáncer, desnutrición, uso de drogas intravenosas, trasplante de órganos, terapias inmunosupresoras y corticoides, traumatismos, cirugías, quemaduras y embarazo. En una revisión sistemática se determinó que las condiciones subyacentes fueron: diabetes mellitus (40\%), malignidades hematológicas (33\%), trasplante de órganos sólidos (14\%), y los factores predisponentes fueron: uso de corticoides (33\%), neutropenia (20\%), quimioterapia (18\%), trauma $(10 \%) .^{3}$

El primer caso de mucormicosis fue descrito en 1885 por Paltauf, quien empleó el término mycosis mucorina (que posteriormente se modificó a mucormicosis) y describió la infección diseminada en un paciente oncológico., 5, 6 En 1943 Gregory y otros hicieron las primeras descripciones de la enfermedad rinocerebral en tres pacientes diabéticos. ${ }^{9}$ En 1957, y debido a lo inusual de la enfermedad, Baker reportó una decena de casos reunidos en 75 años $^{4}$, mientras que en la presente casuística se reunieron cuatro casos en menos de dos meses.

Clínicamente, y según su distribución, la mucormicosis se puede clasificar de la siguiente manera:-6,8,9,11

1. Rinocerebral (39\%): por lo general en primer hay invasión de mucosa nasal o palatina, con progresión a los senos paranasales, expansión a lo largo de los vasos y los nervios, pudiendo alcanzar la órbita, fosa craneal media y el seno cavernoso logrando llegar a las meninges y al cerebro. Se considera que la forma rinoorbitaria y paranasal son estadios tempranos de la forma rinocerebral. En el sistema nervioso central puede ocasionar trombosis séptica del seno cavernoso, 
meningoencefalitis micótica, hemorragias intracerebrales, abscesos, infartos cerebrales, hipertensión endocraneana, coma y muerte. Una costra necrótica sangrante en el paladar o en la mucosa nasal y un drenaje ocular de pus negruzco orientan al diagnóstico, aunque las zonas necróticas en cavidad nasal, paladar o cara son signos iniciales sólo en $50 \%$ de los pacientes.

2. Pulmonar (24\%): por inhalación de esporas del hongo que se depositan en bronquios terminales y alvéolos. La forma pulmonar puede presentarse en pacientes con leucemia y neutropenia.

3. Cutánea (19\%): luego de traumatismo, herida o quemaduras. Pueden cursar con fasciítis necrotizante.

4. Digestivo (7\%): se observa más comúnmente en pacientes con desnutrición calórico-proteica.

5. Sistémica $(6 \%)$ : se caracteriza por presentar vasculitis con fenómenos trombóticos e infartos en varios órganos en los que frecuentemente se sobreagregan infecciones bacterianas. Esta forma se presenta en pacientes con déficit inmunológico grave como trasplantados, leucémicos y nefrópatas tratados con desferroxiamina.

6) Miscelánea.

Las características clínicas dependen de la forma de compromiso, siendo las más frecuentes: ${ }^{2,4,6,8-11}$

A. Sistémicas: fiebre.

B. Rinosinusal: rinorrea, obstrucción nasal, epistaxis, sinusitis (usualmente no responden a la terapia antimicrobiana y los pacientes se deterioran rápidamente), perforación de tabique nasal, ulceración y necrosis nasal, septal, palatina y gingival.

C. Oftálmica: dolor periorbitario, anestesia corneal, proptosis, disminución o pérdida de la visión (en caso de trombosis retiniana), quemosis, celulitis orbitaria, edema periorbitario y facial, defectos pupilares, paresia de músculos extraoculares, oftalmoplegia.

D. Neurológicas: cefalea, diplopía, algia facial, dolor maxilar unilateral, parestesias faciales, parálisis de nervios craneales, obnubilación, La disfunción de nervios craneales, especialmente el V y VII, ocurre de manera tardía manifestando ptosis y midriasis, lo cual es un factor pronóstico grave.

E. Óseas: erosión y destrucción ósea.

En cuanto a la evolución clínica, la forma aguda fulminante se caracteriza por progresión rápida de la enfermedad debido a diseminación vascular del hongo. La forma invasiva crónica, de evolución indolente, puede presentar acumulación de hifas simulando un micetoma. ${ }^{2}$

La microscopía directa, histopatología y cultivos son esenciales para el diagnóstico de mucormicosis..$^{13,14}$

Los mucorales son hongos aerobios, filamentosos, omnipresentes. Están constituidos por hifas anchas, no tabicado con tamaño variable de 10 a 60 micras ramificadas en ángulo abierto (en torno a los $90^{\circ}$ ); existe la posibilidad de confundirlos con Aspergillus spp. pero este es menos grueso y tabicado. ${ }^{4,6}$
La identificación de la especie exacta causante de la mucormicosis no es fácil por la dificultad de aislamiento del hongo en medios de cultivo. Paradójicamente, aun cuando las hifas son vistas en análisis histopatológicos, los cultivos son sólo positivos del 30 al $50 \%$ de casos. ${ }^{8}{ }^{14}$ Las colonias pueden aparecer entre las 12 y 18 horas de la siembra y el crecimiento se completa en 2 a 5 días. ${ }^{9}$ Si el paciente ha recibido anfotericina $\mathrm{B}$, el crecimiento puede ser más lento o estar ausente. ${ }^{2}$

Los signos radiológicos más frecuentes son la osteolisis, engrosamiento nodular de la mucosa del seno y ausencia del nivel del líquido sinusal. ${ }^{11}$

La tomografía axial computarizada de cráneo, revela extensión y profundidad del daño con lesiones orbitales, senos maxilares y etmoidales, con engrosamiento de músculos extraoculares y proptosis, hipertrofia de cornetes, perforación de septum nasal y sinusitis. Además, la presencia de abscesos cerebrales o trombosis de seno cavernoso. ${ }^{4,5,9}$

Los datos histológicos sobresalientes son resultado de la gran afinidad vascular de estos hongos, con invasión y crecimiento en la lámina elástica interna, disección de la media con penetración de hifas al endotelio produciendo trombosis, perforación hemorrágica, infarto y necrosis tisular, hemorragias e inflamación aguda y crónica; tardíamente se involucran venas y vasos linfáticos $(4,9)$.

El tratamiento consta de tres puntos importantes:

Terapia antifúngica temprana: la mucormicosis se consideró fatal hasta que se introdujo la anfotericina B como tratamiento antimicótico en los años 60 del siglo XIX. Los pacientes que son tratados con anfotericina $\mathrm{B}$ tienen 4 veces más probabilidades de sobrevivir. La anfotericina B en suspensiones lipídicas tiene la ventaja de preservar las membranas citoplasmáticas del huésped. La anfotericina B en su formulación lipídica es la terapia de elección y es más efectiva que el deoxicolato, siendo la dosis 3 a $5 \mathrm{mg} / \mathrm{kg} /$ día, por tiempo prolongado, continuando 2 a 3 semanas después de la curación clínica o la dosis sugerida de $5-10 \mathrm{mg} / \mathrm{kg} /$ día en infección del sistema nervioso central. La forma liposomal disminuye la toxicidad renal, además tiene una mejor penetración en los tejidos. Anfotericina B deoxicolato es el tratamiento estándar cuando no hay otras formas disponibles, a dosis de 1 a $1,5 \mathrm{mg} / \mathrm{kg}$ por día, hasta completar una dosis total de 2 a 4 g. La mortalidad a las 12 semanas se duplica si se retrasa el tratamiento 6 o más días. Posaconazol (200 mg 4 veces al día) es el mejor medicamento cuando no se tolera la anfotericina B. Isavuconazol no ofrece ventajas significativas y no incrementa la supervivencia cuando se compara con anfotericina B. La OPS recomienda como primera opción en su fase de inducción (aproximadamente tres semanas) la administración de anfotericina B en formulaciones lipídicas $5 \mathrm{mg} / \mathrm{kg}$ o, ante intolerancia a la anfotericina B, isavuconazozol, dosis de carga $372 \mathrm{mg} /$ EV o VO/por 6 dosis, seguidos de $372 \mathrm{mg} / \mathrm{EV}$ o VO/día. Para la fase de consolidación recomienda isavuconazol, dosis de carga, $372 \mathrm{mg} / \mathrm{EV}$ o VO cada 8 horas por 6 dosis, 
seguidos de $372 \mathrm{mg} / \mathrm{EV}$ o VO/día, hasta mejoría clínica; o, posaconazol. ${ }^{1,2,5,6,8,9,11,13-19}$

Cirugía: practicar la resección y el desbridamiento quirúrgico es crucial y debe realizarse con urgencia y de forma amplia para limitar la propagación de la infección. El debridamiento quirúrgico tiene el objetivo de disminuir la invasión vascular, eliminar tejidos necróticos y facilitar la penetración de antimicóticos. Frecuentemente se requieren varias cirugías para remover el tejido desvitalizado incrementando con esto las posibilidades de éxito terapéutico, por lo tanto, debe realizarse cuantas veces sea necesaria. El desbridamiento extenso, la resección amplia del tejido necrótico blando y hueso, y en algunos casos la evisceración de la órbita es necesaria. La terapia antifúngica asociada a cirugía aumentó significativamente las tasas de supervivencia (69\%), mientras que el $97 \%$ de pacientes sin tratamiento fallecieron $(1,5,6,8,11,13)$.

Manejo y reversión de las comorbilidades que estuvieron descompensadas y que son factor de riesgo de tener la infección (neutropenia, hiperglucemia, acidosis metabólica) $(1,9)$.

El tratamiento antifúngico y quirúrgico agresivo combinado se asocia con una disminución de la tasa de mortalidad a los 90 días; si se administra anfotericina $\mathrm{B}$ en forma tardía se evidencia incremento de la mortalidad hasta $83 \%$. Los factores para un mal pronóstico incluyen afección intracraneal, hemiplejía, hemiparesia, afección de senos paranasales bilateral, úlceras palatinas, necrosis facial e invasión orbitaria (5).

El uso de oxígeno hiperbárico se ha considerado benéfica en algunos pacientes al limitar la extensión de la gangrena $\mathrm{y}$ tejido necrótico; sin embargo, no se recomienda rutinariamente debido a la falta de estudios controlados. ${ }^{9}$ Otros estudios experimentales recomiendan además el uso de bicarbonato de sodio (con insulina) para revertir la cetoacidosis, independientemente que la acidosis sea leve o severa y esto podría asociarse con una mejor evolución de la enfermedad al disminuir la habilidad de los mucorales para invadir tejidos del huésped. ${ }^{14}$

La duración óptima de la terapia no está bien definida y se basa en la respuesta observada en cada paciente, aunque puede tomarse en consideración la resolución de anormalidades radiográficas, cultivos negativos y las biopsias. $^{10}$

Aproximadamente, la mitad de los pacientes con mucormicosis fulminante aguda sobrevive. En casos de mucormicosis crónica, la tasa de sobrevida puede ser del $100 \%{ }^{7}$ En cuanto a las formas clínicas, la diseminada es más letal $(68 \%)$ y la menos severa es la cutánea $(31 \%){ }^{3}$ Cuando se evalúa la mortalidad según terapias recibidas, los pacientes que sólo recibieron tratamiento antifúngico tuvieron una mortalidad de 58\% y aquellos que la combinaron con cirugía tuvieron una tasa de $24 \% .^{12}$

En conclusión, la mucormicosis es una infección micótica rara que se está presentando en mayor número de pacientes diabéticos no controlada por incumplimiento en el tratamiento, por el uso indiscriminado de corticoides y agravado por la falta de una atención oportuna. En los casos presentados ocurridos durante la pandemia COVID-19, el antecedente del uso de corticoides en el tratamiento de la infección por SARS-CoV2 fue un factor de riesgo común. El diagnóstico temprano es de suma importancia para el pronóstico de esta enfermedad. Debe sospecharse en todo paciente con factores de riesgo que presenten fiebre y sinusitis de lenta o nula resolución. Debe hacerse búsqueda activa de escaras y secreciones negruzcas en las fosas nasales y el paladar duro.

El tratamiento debe ser médico y quirúrgico. La mortalidad de esta micosis es de 40 a 50\%, o más, en casos de forma rino-órbito-cerebral o diseminada, aun con tratamiento agresivo

\section{REFERENCIAS BIBLIOGRÁFICAS}

I. Vargas Herrera N, Saavedra Velazco M, Contreras Calero E, Ramos Castillo W, Mendo Urbina F, Pichardo Rodríguez R. Mucormicosis diseminada en un paciente diabético: un reporte de caso. Acta Med Peru. 2019;36(4):287-90.

2. Bertin H. Mucormicosis rinosinusal. Rev. Otorrinolaringol. Cir. Cabeza Cuello. 2003; 63: 122-126.

3. Jeong W, Keighley C,Wolfe R, LeeW, Slavin M et al.The epidemiology and clinical manifestations of mucormycosis: a systematic review and meta-analysis of case reports. Clinical Microbiology and Infection. 2019;25: 26-34.

4. Romero J,BonifazA, Sánchez C, Lagunas A, y Hidalgo H. Mucormicosis rinocerebral. Reporte de doce casos. Rev Med Hosp Gen Mex. 2000; 63 (3): 178- 184.

5. Julián A y Crespo P. Mucormicosis rinocerebral: reporte de un caso y revisión de la literatura. Rev Mex Cir Bucal Maxilofac. 2020; I6 (I): 22-26.

6. Botero J, Moreno L y Caparroso L. Mucormicosis rinocerebral en paciente con cefalea y diabetes mellitus. Asoc Col Infect. 2008; I2(I): $72-6$.

7. Celis E, Burgos A, Villanueva N, Solórzano J, De La Mora A et al. An Emergent Entity: Indolent Mucormycosis of the Paranasal Sinuses. A Multicenter Study. Int Arch Otorhinolaryngol. 2019;23:92100.

8. Aldama A, García J, Aldama F, Insaurralde S, Mendoza de Sánchez G y col. Mucormicosis rinocerebral.An. Fac. Cienc. Méd. (Asunción). 20I7;50(3). http://dx.doi.org/10.18004/anales/20I7.050(03)75-082.

9. Aragonés B, Stolik P, Candelaria E y Veitía S. Mucormicosis ocular. Revista Cubana de Oftalmología. http://scielo.sld.cu/scielo. php?script=sci_arttext\&pid=S0864-2 I 762007000200017.

10. Acosta M, Navarro M,Arévalo My Manrique H. Mucormicosis en un paciente diabético. Rev Soc Peru Med Interna. 20I 0;23: 28-30.

II. Acosta C, Lucio E, Uribe A y Gómez B. Mucormicosis rinocerebral de origen dental, reporte de un caso clínico y revisión de la literatura. Rev Esp Cir Oral Maxilofac. 20।4;3 6(2):68-72.

12. Nucci M, Engelhardt M, Hamed K. Mucormycosis in South America: A review of 143 reported cases. Mycoses. 2019;00:I-9. https ://doi. org/I 0.I I I I/myc. I 2958.

13. Cornely O, Arikan S, Dannaoui E, Grol A, Lagrou K et al. ESCMID and ECMM joint clinical guidelines for the diagnosis and management of mucormycosis 2013. Clin Microbiol Infect. 20I4; 20 (Suppl. 3): 5-26

14. Skiada A, Lass C, Klimko N, Ibrahim A, Roilides E et al. Challenges in the diagnosis and treatment of mucormycosis. Medical Mycology. 2018;56(SuppI I): S93-SI0I.

15. Marty F, Ostrosky L, Cornely O, Mullane K, Perfect J et al. Isavuconazole treatment for mucormycosis: a single-arm openlabel trial and case-control analysis. Lancet Infect Dis. 2016. http:// dx.doi.org//0.1016/SI473-3099(I6)0007|-2.

16. Garcia C. Opciones terapéuticas actuales en las micosis invasoras y papel terapéutico potencial del isavuconazol. Rev Iberoam Micol. 2019;35(4): 192-197. 
17. Maertens J, Raad I, Marr K, Patterson T, Kontoyiannis D et al. Isavuconazole versus voriconazole for primary treatment of invasive mould disease caused by Aspergillus and other filamentous fungi (SECURE): a phase 3, randomised-controlled, non-inferiority trial. www.thelancet.com Published online December 9, 2015 http:// dx.doi.org/I0.1016/S0I40-6736(I5)0II59-9.

18. Bala K, Chander J, Handa U, Singh R and Kumar A. A prospective study of mucormicosis in north India: Experience from a tertiary care hospital. Medical Mycology. 20I5, 53, 248-257
19. OPS. Tratamiento de las enfermedades infecciosas 2020-2022. Octava edición. Organización Panamericana de la Salud, 2019.

\section{CORRESPONDENCIA}

Robert Cumpa

robertcumpa@gmail.com

Fecha de recepción : 25-10-2020.

Fecha de aceptación : |6-|| -2020. 